PROCEEDINGS OF THE

AMERICAN MATHEMATICAL SOCIETY

Volume 129, Number 4, Pages 1005-1014

S 0002-9939(00)05641-0

Article electronically published on October 16, 2000

\title{
SUBSEMIVARIETIES OF $Q$-ALGEBRAS
}

\author{
M. H. FAROUGHI
}

(Communicated by Dale Alspach)

\begin{abstract}
A variety is a class of Banach algebras $V$, for which there exists a family of laws $\left\{\|P\| \leq K_{p}\right\}_{P}$ such that $V$ is precisely the class of all Banach algebras $A$ which satisfies all of the laws (i.e. for all $P,\|P\|_{A} \leq K_{p}$ ). We say that $V$ is an $H$-variety if all of the laws are homogeneous. A semivariety is a class of Banach algebras $W$, for which there exists a family of homogeneous laws $\left\{\|P\| \leq K_{P}\right\}_{P}$ such that $W$ is precisely the class of all Banach algebras $A$, for which there exists $K>0$ such that for all homogeneous polynomials $P,\|P\|_{A} \leq K^{i} \cdot K_{P}$, where $i=\operatorname{deg}(P)$. However, there is no variety between the variety of all $I Q$-algebras and the variety of all $I R$-algebras, which can be defined by homogeneous laws alone. So the theory of semivarieties and the theory of varieties differ significantly. In this paper we shall construct uncountable chains and antichains of semivarieties which are not varieties.
\end{abstract}

\section{INTRODUCTION}

Let $A$ be a Banach algebra and $P\left(X_{1}, \ldots, X_{n}\right)$ a polynomial (in several noncommuting variables without constant). We define

$$
\|P\|_{A}=\sup \left\{\left\|P\left(x_{1}, \ldots, x_{n}\right)\right\|: x_{i} \in A,\left\|x_{i}\right\| \leq 1(1 \leq i \leq n)\right\} .
$$

By a law we mean a formal expression $\|P\| \leq K$ where $K \in R$ and $P$ is a polynomial and we say that $A$ satisfies the above law if $\|P\|_{A} \leq K$. We say that a law is homogeneous if $P$ is homogeneous. A variety is a class of Banach algebras (real or complex) for which there exists a family of laws such that all of its members satisfy all of the laws, or equivalently, a variety is a class of Banach algebras which is closed under taking closed subalgebras, quotient algebras by closed ideals, products, and images under isometric isomorphisms (see [1]).

A semivariety is a class of Banach algebras for which there exists a family of laws $\left\{\|P\| \leq K_{P}\right\}$ such that it is precisely the class of all Banach algebras $A$ for which there are $K, a>0$ such that for all $P,\|P\|_{A, a} \leq K \cdot K_{P}$, where

$$
\|P\|_{A, a}=\sup \left\{\left\|P\left(x_{1}, \ldots, x_{n}\right)\right\|: x_{i} \in A,\left\|x_{i}\right\| \leq a(1 \leq i \leq n)\right\} .
$$

Every semivariety is an $H$-semivariety (can be obtained by homogeneous laws), but not every variety is an $H$-variety ([1] and [2]) which is a significant difference between them.

Let $V$ be a variety and let $\left\{L_{a}\right\}_{a \in I}$ be the family of all families of laws which determines $V$. We define

$$
|P|_{V}=\inf \left\{K: \exists a \in I ;(\|P\| \leq K) \in L_{a}\right\} .
$$

Received by the editors December 11, 1998 and, in revised form, June 10, 1999.

1991 Mathematics Subject Classification. Primary 46H99; Secondary 06B20. 
The family $\left\{|P|_{V}\right\}_{P}$ is a family of laws which determines $V$. By means of the mapping $P \rightarrow|P|_{V}$ we can compare the elements of the lattice of all varieties and the elements of all semivarieties.

1.1. Lemma. Let $V$ and $W$ be two varieties. Then $V \subseteq W$ if and only if for all polynomials $P,|P|_{V} \leq|P|_{W}$.

Proof. See [2].

Each semivariety can be generated by an $H$-variety by means of the operation " $\wedge$ " (if $V$ is a class of Banach algebras, $\hat{V}$ will be the class of all Banach algebras isomorphic to members of $V$ (see [1])).

1.2. Lemma. Let $A$ and $B$ be two semivarieties generated by the $H$-varieties, respectively, $V$ and $W$. Then $A \subseteq B$ if and only if there exists $K>0$, such that for all homogeneous polynomials $P$ of degree $i$,

$$
|P|_{V} \leq K^{i}|P|_{W}
$$

Proof. See [3].

1.3. Lemma. If a variety $V$ is determined by the homogeneous laws

$$
\left\{\|P\| \leq K_{P}\right\}_{p \in L}
$$

where $L$ is a class of homogeneous polynomials, then

$$
\widehat{V}=\left\{A: \exists K>0 ;\|P\|_{A} \leq K^{i} \cdot K_{P} \quad \text { for all } P \in L\right\},
$$

where $i=\operatorname{deg}(P)$.

Proof. See [3].

\section{Generators of SEMivarieties}

2.1. Definition. A variety is called a polynomial identity variety if its elements satisfy a class of algebraic identities.

2.2. Lemma. Each polynomial identity variety is a semivariety.

Proof. See 3].

The following theorem, with Lemma 2.2 shows that the lattice of all semivarieties is small compared with the lattice of all varieties.

2.3. Theorem. Let $D$ be a semivariety. Let $N_{2}$ be the variety of all nilpotent Banach algebras of class 2 (i.e. Banach algebras in which all products vanish). Then:

(i) if $D=N_{2}$, then $N_{2}$ is the only variety which generates $D$;

(ii) if $D \neq N_{2}$, then there exist uncountably many $H$-varieties each of which generates $D$.

Proof. (i) is straightforward.

(ii) Given $0<a<1$, let $D \neq N_{2}$. There is an $H$-variety $V$ such that $D=\widehat{V}$. Let $A$ be a Banach algebra such that $V=V(A)$. Now let $A_{a}$ be the algebra derived from the Banach algebra $A$ by changing the product of $A$ to "o" defined as follows:

$$
x \circ y=a(x y) \quad(x, y \in A) .
$$


Let $V_{a}=H\left(A_{a}\right)$, for all $1>a>0$, where $H\left(A_{a}\right)$ is the $H$-variety determined by the homogeneous laws $\|P\|_{A_{a}} \geq\|P\|$. It is easy to see that for all homogeneous $P$ and all $1>a>0$,

$$
|P|_{V_{a}}=\|P\|_{V\left(A_{a}\right)}=\|P\|_{A_{a}} .
$$

But for all homogeneous $P$ of degree $i,\|P\|_{A_{a}}=a^{i-1}\|P\|_{A}$. So for all homogeneous $P$ and all $1>a>0$,

$$
|P|_{V_{a}}=a^{i-1}\|P\|_{A} .
$$

Therefore, if $1>a>0,1>b>0$ and $a \neq b$, then $V_{a} \neq V_{b}$. Now we shall prove that $D=\widehat{V}_{a}$ for all $1>a>0$. Since $V_{a} \subseteq V, \widehat{V}_{a} \subseteq D$. Now let $B \in D$. By Lemma 1.3 there is $K>0$ such that

$$
\|P\|_{B} \leq K^{i}\|P\|_{A}=K^{i} a^{1-i}\|P\|_{A_{a}} \leq\left(k / a^{2}\right)^{i}\|P\|_{A_{a}}=\left(k / a^{2}\right)^{i}|P|_{V_{a}}
$$

for all homogeneous $P$ of degree $i$. So, $B \in \widehat{V}_{a}$; hence $D \subseteq \widehat{V}_{a}$, which completes the proof of the theorem.

\section{Chains and antichains of subsemivarieties of $Q$-Algebras}

We saw that most of the well-known semivarieties are varieties, and each semivariety has uncountably many generators; however, we shall try to build uncountable chains and antichains of subsemivarieties of $Q$-algebras which are not varieties. (A $Q$-algebra is a Banach algebra which is bicontinuously isomorphic with the quotient of a uniform algebra by a closed ideal, and the class of all $Q$-algebras is a semivariety (see [7]).)

3.1. Definition. Let $I=(0,1)$. We say that the family $\left\{a_{i}\right\}_{i \in I}$ of real decreasing sequences is increasing if for all $i, j \in I$ and all $n \in N, j \geq i$ implies

$$
a_{j}(n) \geq a_{i}(n) .
$$

3.2. Theorem. There exists an uncountable chain of semivarieties which are not varieties.

Proof. Let $I=(0,1)$ and let $\left\{a_{i}\right\}_{i \in I}$ be an increasing family of sequences such that there exists $C>1$, so that for all $i \in I$,

$$
\lim _{n \rightarrow \infty}\left[a_{i}(1) \cdots a_{i}(n) /\left(a_{i}(1)\right)^{n}\right]^{1 / n^{c}}=i .
$$

For each $i \in I$, let $w_{i}(n)=a_{i}(1) \cdots a_{i}(n)$, and let $L_{a_{i}}^{1}$ is the weighted sequence algebra. Suppose for all $i \in I, V_{i}$ is the $H$-variety determined by the laws

$$
\left\|X_{1} \cdots X_{n}\right\| \leq a_{i}(1) \cdots a_{i}(n) /\left(a_{i}(1)\right)^{n} .
$$

Since

$$
\left\|X_{1} \cdots X_{n}\right\|_{L_{a_{i}}^{1}}=a_{i}(1) \cdots a_{i}(n) /\left(a_{i}(1)\right)^{n}
$$

(see 2], Theorem 4.5), it follows that

$$
\left|X_{1} \cdots X_{n}\right|_{V_{i}}=a_{i}(1) \cdots a_{i}(n) /\left(a_{i}(1)\right)^{n} .
$$

Now consider the family $\left\{\widehat{V}_{i}\right\}_{i \in I}$ of semivarieties. First we shall prove that all elements of $\left\{\widehat{V}_{i}\right\}_{i \in I}$ are different. Let $i, j \in I$ and $\widehat{V}_{i}=\widehat{V}_{j}$. Then, there exist $K>0$ and $L>0$ such that for all homogeneous polynomials $P$ of degree $r$,

$$
|P|_{V_{i}} \leq K^{r}|P|_{V_{j}} \text { and } \quad|P|_{V_{j}} \leq L^{r}|P|_{V_{i}} .
$$


Therefore for all $n \geq 1$,

$$
a_{i}(1) \cdots a_{i}(n) /\left(a_{i}(1)\right)^{n} \leq K^{n} a_{j}(1) \cdots a_{j}(n) /\left(a_{j}(1)\right)^{n}
$$

and

$$
a_{j}(1) \cdots a_{j}(n) /\left(a_{j}(1)\right)^{n} \leq L^{n} a_{i}(1) \cdots a_{i}(n) /\left(a_{i}(1)\right)^{n} .
$$

Hence

$$
\left[a_{j}(1) / a_{i}(1)\right]^{n}\left[a_{i}(1) \cdots a_{i}(n) / a_{j}(1) \cdots a_{j}(n)\right] \leq K^{n}
$$

and

$$
\left[a_{i}(1) / a_{j}(1)\right]^{n}\left[a_{j}(1) \cdots a_{j}(n) / a_{i}(1) \cdots a_{i}(n)\right] \leq L^{n} .
$$

So by $(*)$, we have

$$
i / j \leq 1 \quad \text { and } \quad j / i \leq 1
$$

Thus, $i=j$, so the elements of $\left\{\widehat{V}_{i}\right\}$ are different.

Now we shall prove that, for each $i \in I, \widehat{V}_{i}$ is not a variety. Let $i \in I$ and $m \geq 1$. Let for all $n \geq 1$,

$$
a_{i}^{m}(n)= \begin{cases}1 & \text { if } m \geq n \\ a_{i}(1) \cdots a_{i}(n) /\left(a_{i}(1)\right)^{n} & \text { otherwise. }\end{cases}
$$

Let $a_{i}^{m}=\left(a_{i}^{m}(n)\right)_{n=1}^{\infty}$. Then $a_{i}^{m}$ is a decreasing positive sequence of real numbers. Let

$$
A_{i}^{m}=L_{a_{i}^{m}}^{1}
$$

Then for all $n \geq 1$,

$$
\left\|X_{1} \cdots X_{n}\right\|_{A_{i}^{m}}=a_{i}^{m}(1) \cdots a_{i}^{m}(n) /\left(a_{i}^{m}(1)\right)^{n} .
$$

However, $a_{i}^{m}(1)=1$, so for all $n \geq 1$,

$$
\left\|X_{1} \cdots X_{n}\right\|_{A_{i}^{m}}=a_{i}^{m}(2) \cdots a_{i}^{m}(n) .
$$

It is clear that if $m \geq n$, then

$$
\left\|X_{1} \cdots X_{n}\right\|_{A_{i}^{m}}=1
$$

Now let $m<n$. Then we have

$(* * *) \quad\left\|X_{1} \cdots X_{n}\right\|_{A_{i}^{m}}=a_{i}^{m}(2) \cdots a_{i}^{m}(n) \leq a_{i}^{m}(n)=a_{i}(1) \cdots a_{i}(n) /\left(a_{i}(1)\right)^{n}$.

Now let

$$
L=\left[a_{i}(1) \cdots a_{i}(m) /\left(a_{i}(1)\right)^{m}\right]^{-1 / m} .
$$

Next we shall prove that for all $n \geq 1$,

$$
\left\|X_{1} \cdots X_{n}\right\|_{A_{i}^{m}} \leq L^{n} a_{i}(1) \cdots a_{i}(n) /\left(a_{i}(1)\right)^{n} .
$$

If $m \geq n$, then we have

$$
\left[a_{i}(1) \cdots a_{i}(m) /\left(a_{i}(1)\right)^{m}\right]^{n} \leq\left[a_{i}(1) \cdots a_{i}(n) /\left(a_{i}(1)\right)^{n}\right]^{m} .
$$

So by $(* *)$, we have

$$
\begin{aligned}
\left\|X_{1} \cdots X_{n}\right\|_{A_{i}^{m}} & =1 \leq\left[a_{i}(1) \cdots a_{i}(m) /\left(a_{i}(1)\right)^{m}\right]^{-n / m} \cdot\left[a_{i}(1) \cdots a_{i}(n) /\left(a_{i}(1)\right)^{n}\right] \\
& =L^{n} a_{i}(1) \cdots a_{i}(n) /\left(a_{i}(1)\right)^{n} .
\end{aligned}
$$


Now let $m<n$. Since $L \geq 1$, by $(* * *)$ we have

$$
\begin{aligned}
\left\|X_{1} \cdots X_{n}\right\|_{A_{i}^{m}} & \leq a_{i}(1) \cdots a_{i}(n) /\left(a_{i}(1)\right)^{n} \\
& \leq L^{n} a_{i}(1) \cdots a_{i}(n) /\left(a_{i}(1)\right)^{n},
\end{aligned}
$$

so that for all $i \in I$ and all $m \geq 1$,

$$
A_{i}^{m} \in \widehat{V}_{i}
$$

Now we shall prove that for all $i \in I$,

$$
\prod_{m=1}^{\infty} A_{i}^{m} \notin \widehat{V}_{i}
$$

Since for all $n \geq 1$

$$
\left\|X_{1} \cdots X_{n}\right\|_{\Pi A_{i}^{m}}=\sup _{m \geq 1}\left\|X_{1} \cdots X_{n}\right\|_{A_{i}^{m}}
$$

(for a proof see [3]), so by $(* *)$ we have

$$
\left\|X_{1} \cdots X_{n}\right\|_{\Pi A_{i}^{m}}=1 \text {. }
$$

Now let $\prod_{m=1}^{\infty} A_{i}^{m} \in \widehat{V}_{i}$. So there exists $M>0$ such that for all $n \geq 1$,

$$
M^{n} a_{i}(1) \cdots a_{i}(n) /\left(a_{i}(1)\right)^{n} \geq 1 .
$$

So by $(*), i \geq 1$, which is a contradiction, so that for all $i \in I, \prod_{m=1}^{\infty} A_{i}^{m} \notin \widehat{V}_{i}$. Therefore for all $i \in I, \widehat{V}_{i}$ is not a variety.

The family $\left\{\widehat{V}_{i}\right\}$ is also a chain, because if $i, j \in I$ and $j>i$, we take $L=$ $a_{j}(1) / a_{i}(1)$. Then for all $n \geq 1$,

$$
\left|X_{1} \cdots X_{n}\right|_{V_{i}} \leq L^{n}\left|X_{1} \cdots X_{n}\right|_{V_{j}}
$$

so $\widehat{V}_{i} \subseteq \widehat{V}_{j}$.

It remains to show that there is an increasing family $\left\{a_{i}\right\}_{i \in I}$ of sequences which also satisfies $(*)$. For example, let $I=(0,1)$ and for each $i \in I$, let

$$
a_{i}=\left\{i^{2 k-2}\right\}_{k=1}^{\infty} \text {. }
$$

Then $\left\{a_{i}\right\}$ is an increasing family of sequences. Let $C=2$. We have

$$
\lim _{n \rightarrow \infty}\left[a_{i}(1) \cdots a_{i}(n) /\left(a_{i}(1)\right)^{n}\right]^{1 / n^{2}}=\lim _{n \rightarrow \infty}\left(i \cdot i^{2} \cdots i^{n-1}\right)^{2 / n^{2}}=i .
$$

3.3. Remark. In Theorem 3.2, for each $i \in I, V_{i}$ is a non-polynomial identity variety (see Lemma 2.2), so there exists an uncountable tower of varieties whose elements are non-polynomial identity varieties.

3.4. Theorem. There exists an uncountable antichain of semivarieties which are not varieties.

Proof. Let $i>1$. Let for each $n \geq 1$

$$
C_{n}^{i}= \begin{cases}n 2^{n} & \text { if there is } m \geq 1 \text { such that }(2 m+1)^{i}>n \geq(2 m)^{i}, \\ -n 2^{n} & \text { if there is } m \geq 1 \text { such that }(2 m)^{i}>n \geq(2 m-1)^{i} .\end{cases}
$$

Let $i>1, j>1$ and $j>i$. We have

$$
\lim _{m \rightarrow \infty}\left[(2 m)^{i}-(2 m-1)^{i}\right]=\lim _{m \rightarrow \infty}\left[(2 m+1)^{j / i}-(2 m)^{j / i}\right]=\infty,
$$


so there are $N_{1}>0$ and $N_{2}>0$ such that

$$
m^{\prime} \geq N_{1} \Rightarrow\left(2 m^{\prime}\right)^{i}-\left(2 m^{\prime}-1\right)^{i}>4
$$

and

$$
m \geq N_{2} \Rightarrow(2 m+1)^{j / i}-(2 m)^{j / i}>4 .
$$

Let $N=\max \left(N_{1}, N_{2}\right)$. Let $n>N$; then there is $N_{3}$ such that

$$
m^{\prime \prime} \geq N_{3} \Rightarrow\left(2 m^{\prime \prime}\right)^{j}>n .
$$

Let $m_{0}=\max \left(N, N_{3}\right)$. Since $m_{0} \geq N_{3}$,

$$
\left(2 m_{0}\right)^{j}>n \text {. }
$$

Since $m_{0} \geq N, m_{0} \geq N_{2}$. Hence

$$
\left(2 m_{0}+1\right)^{j / i}-\left(2 m_{0}\right)^{j / i}>4,
$$

so there is $m_{0}^{\prime}$ such that

$$
\left(2 m_{0}+1\right)^{j / i}>2 m_{0}^{\prime}>2 m_{0}^{\prime}-1>\left(2 m_{0}\right)^{j / i}
$$

or

$$
\left(2 m_{0}+1\right)^{j}>\left(2 m_{0}^{\prime}\right)^{i}>\left(2 m_{0}^{\prime}-1\right)^{i}>\left(2 m_{0}\right)^{j} .
$$

But we have

$$
m_{0}^{\prime}>\left[\left(2 m_{0}\right)^{j / i}+1\right] / 2 \geq m_{0} \geq N \geq N_{1},
$$

so there is $n_{0}$ such that

$$
\left(2 m_{0}^{\prime}\right)^{i}>n_{0}>n_{0}-1>\left(2 m_{0}^{\prime}-1\right)^{i} .
$$

Therefore, by (11), (21) and (3), there exist $m_{0}, m_{0}^{\prime}$ and $n_{0}$ such that

$$
\left(2 m_{0}+1\right)^{j}>\left(2 m_{0}^{\prime}\right)^{i}>n_{0}>n_{0}-1>\left(2 m_{0}^{\prime}-1\right)^{i}>\left(2 m_{0}\right)^{j}>n .
$$

We have

$$
\begin{aligned}
\sum_{k=1}^{n_{0}}\left(C_{k}^{j}-C_{k}^{i}\right) & =\sum_{k=1}^{n_{0}-2}\left(C_{k}^{j}-C_{k}^{i}\right)+C_{n_{0}-1}^{j}+C_{n_{0}}^{j}-C_{n_{0}-1}^{i}-C_{n_{0}}^{i} \\
& \geq-\sum_{k=1}^{n_{0}-2} 2 k 2^{k}+C_{n_{0}-1}^{j}+C_{n_{0}}^{j}-C_{n_{0}-1}^{i}-C_{n_{0}}^{i} \\
& \geq-\left(n_{0}-1\right)\left(2^{n_{0}}-4\right)+C_{n_{0}-1}^{j}+C_{n_{0}}^{j}-C_{n_{0}-1}^{i}-C_{n_{0}}^{i} .
\end{aligned}
$$

By (4), we have

$$
\begin{aligned}
& C_{n_{0}-1}^{j}=\left(n_{0}-1\right) 2^{n_{0}-1}, \quad C_{n_{0}}^{j}=n_{0} 2^{n_{0}}, \\
& C_{n_{0}-1}^{i}=-\left(n_{0}-1\right) 2^{n_{0}-1}, \quad C_{n_{0}}^{i}=-n_{0} 2^{n_{0}},
\end{aligned}
$$

so that

$$
\begin{aligned}
\sum_{k=1}^{n_{0}}\left(C_{k}^{j}-C_{k}^{i}\right) \geq- & \left(n_{0}-1\right)\left(2^{n_{0}}-4\right)+\left(n_{0}-1\right) 2^{n_{0}-1}+n_{0} 2^{n_{0}} \\
& +\left(n_{0}-1\right) 2^{n_{0}-1}+n_{0} 2^{n_{0}}=n_{0} 2^{n_{0}+1}+4\left(n_{0}-1\right) .
\end{aligned}
$$

Thus

$$
1 / n_{0} \sum_{k=1}^{n_{0}}\left(C_{k}^{j}-C_{k}^{i}\right) \geq 2^{n_{0}+1}+4\left(n_{0}-1\right) / n_{0} \geq 2^{n_{0}+1} \geq 2^{n+1},
$$


so that for all $i>1, j>1$ and $j>i$,

$$
\sup _{n>1} 1 / n \sum_{k=1}^{n}\left(C_{k}^{j}-C_{k}^{i}\right)=\infty
$$

Now let $i>1, j>1$ and $j>i$. There are $M_{1}>0$ and $M_{2}>0$ such that

$$
m^{\prime} \geq M_{1} \Rightarrow\left(2 m^{\prime}+1\right)^{i}-\left(2 m^{\prime}\right)^{i}>4
$$

and

$$
m \geq M_{2} \Rightarrow(2 m+2)^{j / i}-(2 m+1)^{j / i}>4 .
$$

Let $M=\max \left(M_{1}, M_{2}\right)$ and let $n>M$; then there is $M_{3}>0$ such that

$$
m^{\prime \prime} \geq M_{3} \Rightarrow\left(2 m^{\prime \prime}+1\right)^{j}>n .
$$

Let $m_{1}=\max \left(M_{1}, M_{3}\right)$. Since $m_{1} \geq M_{3}$,

$$
\left(2 m_{1}+1\right)^{j}>n
$$

Also, since $m_{1} \geq M, m_{1} \geq M_{2}$. Hence

$$
\left(2 m_{1}+2\right)^{j / i}-\left(2 m_{1}+1\right)^{j / i} \geq 4 .
$$

There is $m_{1}^{\prime}$ such that

$$
\left(2 m_{1}+2\right)^{j}>\left(2 m_{1}^{\prime}+1\right)^{i}>\left(2 m_{1}^{\prime}\right)^{i}>\left(2 m_{1}+1\right)^{j} .
$$

But, we have

$$
m_{1}^{\prime} \geq\left[\left(2 m_{1}+1\right)\right]^{j / i} / 2 \geq m_{1} \geq M_{1},
$$

so there is $n_{1}$ such that

$$
\left(2 m_{1}^{\prime}+1\right)^{i}>n_{1}>n_{1}-1>\left(2 m_{1}^{\prime}\right)^{i} .
$$

Therefore, by (6), (7) and (8), there exist $m_{1}, m_{1}^{\prime}$ and $n_{1}$ such that

(9) $\quad\left(2 m_{1}+2\right)^{j}>\left(2 m_{1}^{\prime}+1\right)^{i}>n_{1}>n_{1}-1>\left(2 m_{1}^{\prime}\right)^{i}>\left(2 m_{1}+1\right)^{j}>n$.

We have

$$
\begin{aligned}
\sum_{k=1}^{n_{1}}\left(C_{k}^{j}-C_{k}^{i}\right) & \leq \sum_{k=1}^{n_{1}} 2 k 2^{k}+C_{n_{1}-1}^{j}+C_{n_{1}}^{j}-C_{n_{1}-1}^{i}-C_{n_{1}}^{i} \\
& \leq\left(n_{1}-1\right)\left(2^{n_{1}}-4\right)+C_{n_{1}-1}^{j}+C_{n_{1}}^{j}-C_{n_{1}-1}^{i}-C_{n_{1}}^{i} .
\end{aligned}
$$

By (9), we have

$$
\begin{aligned}
& C_{n_{1-1}}^{j}=-\left(n_{1}-1\right) 2^{n_{1}-1}, \quad C_{n_{1}}^{j}=-n_{1} 2^{n_{1}}, \\
& C_{n_{1}-1}^{i}=\left(n_{1}-1\right) 2^{n_{1}-1}, \quad C_{n_{1}}^{i}=n_{1} 2^{n_{1}}
\end{aligned}
$$

so that

$$
\begin{aligned}
\sum_{k=1}^{n_{1}}\left(C_{k}^{j}-C_{k}^{i}\right) & \leq\left(n_{1}-1\right)\left(2^{n_{1}}-4\right)-\left(n_{1}-1\right) 2^{n_{1}-1}-n_{1} 2^{n_{1}}-\left(n_{1}-1\right) 2^{n_{1}-1}-n_{1} 2^{n_{1}} \\
& =-n_{1} 2^{n_{1}+1}-4\left(n_{1}-1\right) .
\end{aligned}
$$

Thus

$$
1 / n_{1} \sum_{k=1}^{n_{1}}\left(C_{k}^{j}-C_{k}^{i}\right) \leq-2^{n_{1}+1}-\left[4\left(n_{1}-1\right)\right] / n_{1} \leq-2^{n_{1}+1} \leq-2^{n+1},
$$


so that, for all $i>1, j>1$ and $j>i$,

$$
\inf _{n \geq 1} 1 / n \sum_{k=1}^{n}\left(C_{k}^{j}-C_{k}^{i}\right)=-\infty .
$$

By (5) and (10), we conclude that for all $i>1, j>1$ and $i \neq j$,

$$
\sup _{n \geq 1} 1 / n \sum_{k=1}^{n}\left(C_{k}^{j}-C_{k}^{i}\right)=+\infty .
$$

Now let for all $i>1$ and all $n>1\left(a_{i}(1)=1\right)$,

$$
a_{i}(n)=\exp \left[-\left(n 2^{2 n}+C_{n}^{i}\right)\right]
$$

Then for all $i>1,\left\{a_{i}(n)\right\}_{n \geq 1}$ is a decreasing sequence of positive real numbers. Now let for all $i>1, V_{i}$ be the variety determined by the laws

$$
\left\|X_{1} \cdots X_{n}\right\| \leq a_{i}(1) \cdots a_{i}(n) /\left(a_{i}(1)\right)^{n} \quad(n \geq 1) .
$$

Since

$$
\left\|X_{1} \cdots X_{n}\right\|_{L_{a_{i}}^{1}}=a_{i}(1) \cdots a_{i}(n) /\left(a_{i}(1)\right)^{n}
$$

it follows that for all $n \geq 1$ and all $i>1$,

$$
\left|X_{1} \cdots X_{n}\right|_{V_{i}}=a_{i}(1) \cdots a_{i}(n) /\left(a_{i}(1)\right)^{n} .
$$

For all $i>1, j>1$ and $i \neq j$ we have

$$
\begin{aligned}
\sup _{n \geq 1}\left[a_{i}(1) \cdots a_{i}(n) / a_{j}(1) \cdots a_{j}(n)\right]^{1 / n} & \\
= & \sup _{n \geq 1}\left\{\exp \left[\sum_{k=1}^{n}\left(-k 2^{2 k}-C_{k}^{i}\right)\right] / \exp \left[\sum_{k=1}^{n}\left(-k 2^{2 k}-C_{k}^{j}\right)\right]\right\}^{1 / n} \\
= & \sup _{n \geq 1}\left[\exp 1 / n \sum_{k=1}^{n}\left(C_{k}^{j}-C_{k}^{i}\right)\right]=\exp \sup _{n \geq 1} 1 / n \sum_{k=1}^{n}\left(C_{k}^{j}-C_{k}^{i}\right) .
\end{aligned}
$$

So by (11), we have

$$
\sup \left[a_{i}(1) \cdots a_{i}(n) /\left(a_{j}(1) \cdots a_{j}(n)\right]^{1 / n}=+\infty .\right.
$$

Now we shall prove that $\left\{\widehat{V}_{i}\right\}_{i>1}$ is an uncountable antichain of semivarieties whose elements are not varieties. Let $i>1, j>1$ and $i \neq j$. Then by (13), for all $K>0$ and all $L>0$ there exist $m$ and $n$ such that

$$
\left[\left(a_{j}(1) \cdots a_{j}(m)\right) /\left(a_{i}(1) \cdots a_{i}(m)\right)\right]^{1 / m}>K
$$

and

$$
\left[\left(a_{i}(1) \cdots a_{i}(n)\right) /\left(a_{j}(1) \cdots a_{j}(n)\right)\right]^{1 / n}>L .
$$

But for all $i>1$ and all $j>1, a_{i}(1)=a_{j}(1)$, so for all $K>0$ and all $L>0$ there exist $m$ and $n$ such that

$$
\left[a_{j}(1) \cdots a_{j}(m)\right] /\left(a_{j}(1)\right)^{m}>K^{m}\left[a_{i}(1) \cdots a_{i}(m)\right] /\left(a_{i}(1)\right)^{m}
$$

and

$$
\left[a_{i}(1) \cdots a_{i}(n)\right] /\left(a_{i}(1)\right)^{n}>L^{n}\left[a_{j}(1) \cdots a_{j}(n)\right] /\left(a_{j}(1)\right)^{n} .
$$

Thus, by (12), for all $K>0$ and all $L>0$, there exist $m$ and $n$ such that

$$
\left|X_{1} \cdots X_{m}\right|_{V_{j}}>K^{m}\left|X_{1} \cdots X_{m}\right|_{V_{i}}
$$


and

$$
\left|X_{1} \cdots X_{n}\right|_{V_{i}}>L^{n}\left|X_{1} \cdots X_{n}\right|_{V_{j}} .
$$

Therefore, $\widehat{V}_{i} \not \subset \widehat{V}_{j}$ and $\widehat{V}_{j} \not \subset \widehat{V}_{i}$.

Finally, we shall show that each element of $\left\{\widehat{V}_{i}\right\}$ is not a variety. For each $i>1$ and all $m \geq 1$, let

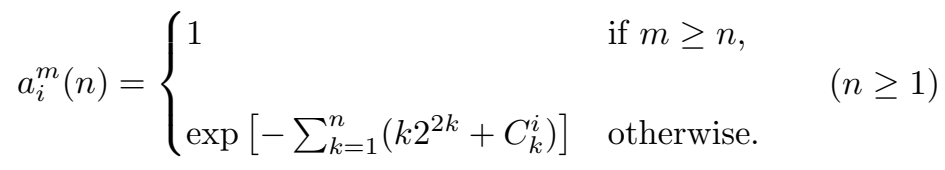

Then $\left\{a_{i}^{m}(n)\right\}_{n \geq 1}$ is a decreasing sequence of positive real numbers. Let for all $i>1$ and all $m \geq 1, A_{i}^{m}=L_{a_{i}^{m}}^{1}$. Then for all $i>1, m \geq 1$ and $n \geq 1$,

$$
\left\|X_{1} \cdots X_{n}\right\|_{A_{i}^{m}}=\left[a_{i}^{m}(1) \cdots a_{i}^{m}(n)\right] /\left(a_{i}^{m}(1)\right)^{n} .
$$

So that if $m \geq n$, then

$$
\left\|X_{1} \cdots X_{n}\right\|_{A_{i}^{m}}=1
$$

and if $n>m$, then

$$
\left\|X_{1} \cdots X_{n}\right\|_{A_{i}^{m}} \leq a_{i}^{m}(n) /\left(a_{i}^{m}(1)\right)^{n}=\exp \left[-\sum_{k=1}^{n}\left(k 2^{2 k}+C_{k}^{i}\right)\right] .
$$

Now let for all $m \geq 1, L_{m}=\exp \left(2^{2 m+2}+2^{m+1}\right)$. Let $i>1, m \geq 1$ and $n \geq 1$. If $m \geq n$, then

$$
\begin{aligned}
& L_{m}^{n}\left\{\exp \left[-\sum_{k=1}^{n}\left(k 2^{2 k}+C_{k}^{i}\right)\right]=\exp \left[n 2^{2 m+2}+n 2^{m+1}-\sum_{k=1}^{n}\left(k 2^{2 k}+C_{k}^{i}\right)\right]\right\} \\
& \quad \geq \exp \left[n 2^{2 m+2}+n 2^{m+1}-\sum_{k=1}^{n}\left(k 2^{2 k}+k 2^{k}\right)\right] \\
& \quad \geq \exp \left[n 2^{2 m+2}+n 2^{m+1}-n \cdot \sum_{k=1}^{m}\left(2^{2 k}+2^{k}\right)\right] \\
& \quad=\exp \left[n 2^{2 m+2}+n 2^{m+1}-n\left(2^{2 m+2}-4\right) / 3-n\left(2^{m+1}-2\right)\right] \\
& \quad=\exp \left[(2 n / 3) 2^{2 m+2}+(10 / 3) n\right] \geq 1=\left\|X_{1} \cdots X_{n}\right\|_{A_{i}^{m}} .
\end{aligned}
$$

Now let $n>m$. Since for all $m \geq 1, L_{m} \geq 1$,

$$
\left\|X_{1} \cdots X_{n}\right\|_{A_{i}^{m}} \leq \exp \left[-\sum_{k=1}^{n}\left(k 2^{2 k}+C_{k}^{i}\right)\right] \leq L_{m}^{n} \exp \left[-\sum_{k=1}^{n}\left(k 2^{2 k}+C_{k}^{i}\right)\right] .
$$

Thus, for all $i>1, m \geq 1$ and $n \geq 1$

$$
\begin{aligned}
\left\|X_{1} \cdots X_{n}\right\|_{A_{i}^{m}} & \leq L_{m}^{n} \exp \left[-\sum_{k=1}^{n}\left(k 2^{2 k}+C_{k}^{i}\right)\right]=L_{m}^{n}\left[a_{i}(1) \cdots a_{i}(n)\right] /\left(a_{i}(1)\right)^{n} \\
& =L_{m}^{n}\left|X_{1} \cdots X_{n}\right|_{V_{i}},
\end{aligned}
$$


so that for all $i>1$ and all $m \geq 1, A_{i}^{m} \in \widehat{V}_{i}$. We have $\left\|X_{1} \cdots X_{n}\right\|_{\prod_{m} A_{i}^{m}}=$ $\sup \left\|X_{1} \cdots X_{n}\right\|_{A_{1}^{m}}=1$. Let $L>0$ be such that for all $n \geq 1$,

$$
\left\|X_{1} \cdots X_{n}\right\|_{\prod_{m} A_{i}^{m}} \leq L^{n} \exp \left[-\sum_{k=1}^{n}\left(k 2^{2 k}+C_{k}^{i}\right)\right] .
$$

Thus we have

$$
L^{n} \exp \left[-\sum_{k=1}^{n}\left(k 2^{2 k}+C_{k}^{i}\right)\right] \leq L^{n} \exp \left[-\left(n 2^{2 n}+C_{n}^{i}\right)\right] \leq L^{n} \exp \left[-\left(n 2^{2 n}-n 2^{n}\right)\right] .
$$

For all $n \geq 1$,

$$
L \cdot \exp \left(2^{n}-2^{2 n}\right) \geq 1
$$

which is a contradiction, so for all $i>1, \prod_{m} A_{i}^{m} \notin \widehat{V}_{i}$. Therefore, for all $i>1, \widehat{V}_{i}$ is not a variety. Thus $\left\{\widehat{V}_{i}\right\}_{i>1}$ is an uncountable antichain of semivarieties which are not varieties.

3.5. Remark. In Theorems 3.2 and 3.4, all of the weighted sequence algebras are $Q$-algebras (see [9], Theorem 5.3), so by considering the intersections of the constructed semivarieties with the semivariety of all $Q$-algebras, we conclude that: There are uncountable chain and antichain of subsemivarieties of $Q$-algebras which are not varieties.

3.6. Corollary. There are uncountable chain and antichain of subvarieties of IQalgebras which are not semivarieties.

Proof. Consider that, for all varieties $U$ and $W, \widehat{U \cap W}=\widehat{U} \cap \widehat{W}$.

\section{REFERENCES}

1. P. G. Dixon, Varieties of Banach algebras, Quart. Math. Oxford Ser. (2) 27 (1976), 481-487. MR 55:8794

2. M. H. Faroughi, Uncountable chains and antichains of varieties of Banach algebras, J. Math. Analysis and Applications 168 (1992), 184-194. MR 93f:46113

3. M. H. Faroughi, Semivarieties of Banach algebras, to appear.

4. F. F. Bonsall, J. Duncan, Complete Normed Algebras, Springer-Verlag, Berlin, 1973. MR 54:11013

5. Hanna Neumann, Varieties of groups, Springer-Verlag, Berlin, Heidelberg, New York, 1967. MR 35:6734

6. Sidney A. Morris, Varieties of topological groups, Bull. Austral. Math. Soc. 1 (1969), 145-160. MR 41:3655a

7. Walter Taylor, Varieties of topological algebras, J. Austral. Math. Soc. 23 (1977), 207-241. MR 56:5392

8. P. G. Dixon, A characterization of closed subalgebras of $B(H)$, Proc. Edinburgh Math. Soc. 20 (1976-77), 215-217. MR 55:11070

9. Sidney J. Diestel, A. Morris and Stephen A. Saxon, Varieties of locally convex topological vector spaces, Bull. Amer. Math. Soc. 77 (1971), 799-803. MR 43:7901

10. P. G. Dixon, Radical Q-algebras, Glasgow Math. J. 17 (1976), 119-126. MR 53:14136

Department of Pure Mathematics, University of Tabriz, Tabriz, Iran

E-mail address: mhfaroughi@ark.tabrizu.ac.ir 